\title{
Does Polyethylene Glycol (PEG) Plus Ascorbic Acid Induce More Mucosal Injuries than Split-Dose 4-L PEG during Bowel Preparation?
}

Min Sung Kim*, Jongha Park*, Jae hyun Park*, Hyung Jun Kim*, Hyun Jeong Jang*, Hee Rin Joo*, Ji Yeon Kim, Joon Hyuk Choi*, Nae Yun Heo*, Seung Ha Park*, Tae Oh Kim*, and Sung Yeon Yang*

Departments of *Internal Medicine and ${ }^{\dagger}$ Pathology, Haeundae Paik Hospital, Inje University School of Medicine, Busan, Korea

\section{See editorial on page 160.}

Background/Aims: The aims of this study were to compare the bowel-cleansing efficacy, patient affinity for the preparation solution, and mucosal injury between a split dose of polyethylene glycol (SD-PEG) and low-volume PEG plus ascorbic acid (LV-PEG+Asc) in outpatient scheduled colonoscopies. Methods: Of the 319 patients, 160 were enrolled for SDPEG, and 159 for LV-PEG+Asc. The bowel-cleansing efficacy was rated according to the Ottawa bowel preparation scale. Patient affinity for the preparation solution was assessed using a questionnaire. All mucosal injuries observed during colonoscopy were biopsied and histopathologically reviewed. Results: There was no significant difference in bowel cleansing between the groups. The LV-PEG+Asc group reported better patient acceptance and preference. There were no significant differences in the incidence or characteristics of the mucosal injuries between the two groups. Conclusions: Compared with SD-PEG, LV-PEG+Asc exhibited equivalent bowel-cleansing efficacy and resulted in improved patient acceptance and preference. There was no significant difference in mucosal injury between SD-PEG and LV-PEG+Asc. Thus, the LV-PEG+Asc preparation could be used more effectively and easily for routine colonoscopies without risking significant mucosal injury. (Gut Liver 2016;10:237-243)

Key Words: Colonoscopy; Bowel preparation; Polyethylene glycols; Ascorbic acid; Mucosal injury

\section{INTRODUCTION}

Colonoscopy is the most effective screening method for colorectal disease, but an adequate assessment requires sufficient bowel preparation. ${ }^{1,2}$ Polyethylene glycol (PEG) is widely used for bowel cleansing before colonoscopy because of its effectiveness and acceptable safety. ${ }^{3,4}$ However, PEG can lead to patient discomfort and noncompliance. The 4-L quantity is too large for many patients, and the preparation does not taste good, both of which could lead to insufficient colon cleansing. ${ }^{5-7}$ To combat this effect, several efforts have been made to reduce patient discomfort and to increase compliance with the bowel preparation methods. Recently, low-volume PEG plus ascorbic acid (LV-PEG+Asc) and sodium picosulfate solutions have been developed and have shown better compliance with a noninferior cleansing efficiency compared to PEG. ${ }^{8-11}$ LV-PEG+Asc is the combination of low-dose PEG and vitamin $C$ and can be used as an osmotic laxative; however, it has been well established that sodium phosphate $(\mathrm{NaP})$ and sodium picosulfate (Pico) may cause mucosal injury through osmotic laxative effects. ${ }^{12-14}$ Thus, there is a possibility of mucosal damage to the colon from the use of Asc. The efficacy of bowel cleansing must be balanced against any other colonic mucosal effects. To the best of our knowledge, there has been no study comparing LV-PEG+Asc with split dose of PEG (SD-PEG) for mucosal injury. Therefore, we aimed to compare the rate of mucosal injury, efficacy and patient affinity for the preparation solution between SD-PEG and LV-PEG+Asc for outpatients scheduled for colonoscopies.

\section{MATERIALS AND METHODS}

\section{Inclusion and exclusion criteria}

This prospective, randomized and investigator-blinded study took place between September 2013 and January 2014. All consecutive outpatients or patients visiting the Health Screening Center between the ages of 20 and 65 years who were scheduled

Correspondence to: Jongha Park

Department of Internal Medicine, Haeundae Paik Hospital, Inje University School of Medicine, 875 Haeun-daero, Haeundae-gu, Busan 612-896, Korea Tel: +82-51-797-0200, Fax: +82-51-797-0941, E-mail: neakker@gmail.com

Received on November 12, 2014. Revised on February 9, 2015. Accepted on March 8, 2015. Published online August 11 , 2015 pISSN 1976-2283 eISSN 2005-1212 http://dx.doi.org/10.5009/gnl14439

@ This is an Open Access article distributed under the terms of the Creative Commons Attribution Non-Commercial License (http://creativecommons.org/licenses/by-nc/4.0) which permits unrestricted non-commercial use, distribution, and reproduction in any medium, provided the original work is properly cited. 
to undergo colonoscopy were eligible. Exclusion criteria were the following: age under 20 years or above 65 years; lactation; pregnancy; bowel obstruction; structural intestinal disorders; gut dysmotility; presence of renal failure, congestive heart failure, or liver failure; electrolyte abnormality; inflammatory bowel disease; taking nonsteroidal anti-inflammatory drugs (NSAIDs); obvious lower gastrointestinal symptom (hematochezia, diarrhea, and so forth); and inability to provide informed consent. All patients provided written informed consent. This study was approved by the Institutional Review Board. The protocol was registered on ClinicalTrials.gov (identifier: NCT01964417).

\section{Randomization and bowel preparation protocol}

All eligible patients were randomly assigned to ingest one of the two preparations. The patients were randomized using random number generation (Generator pro 1.69; Segobit Software) to receive SD-PEG or LV-PEG+Asc. The SD-PEG group ingested $2 \mathrm{~L}$ of PEG at 6:00 PM on the day before the procedure and the remaining $2 \mathrm{~L}$ in the morning at least 5 hours before the procedure. Patients were instructed to take PEG $250 \mathrm{~mL}$ every 15 minutes. The LV-PEG+Asc group ingested $1 \mathrm{~L}$ of LV-PEG+Asc at 6:00 PM on the day before the procedure and the remaining $1 \mathrm{~L}$ in the morning at least 5 hours prior to the procedure. Patients were instructed to take LV-PEG+Asc $250 \mathrm{~mL}$ every 15 minutes and to drink at least $1 \mathrm{~L}$ of additional clear liquids. On the day before the colonoscopy, the patients had a soft diet for dinner, and they were allowed clear liquids until 2 hours before the procedure.

\section{Patient affinity for the preparation solution and adverse events}

All of the patients filled in a questionnaire evaluating their compliance, acceptance, preference to repeat the same regimen, and adverse events. This questionnaire was filled out by each patient 30 minutes before the procedure. The compliance was rated using a three-grade scale based on the amount of bowel preparation taken, as follows: 1 , optimal $(\geq 75 \%$ of the prescribed solution); 2, good (75\%-50\% of the prescribed solution); and 3 , poor $(\leq 50 \%$ of the prescribed solution). The acceptance of the preparation was measured if difficulties ingesting the two regimens were encountered (three point scale: 1, easy; 2, tolerable; 3, difficult). The preference was assessed according to the patient's choice of repeating the same regimen for a future colonoscopy. Adverse effects (abdominal pain and discomfort, nausea, vomiting, headache, dizziness, and sleep disturbances) were also systematically collected.

\section{Preparation efficacy and mucosal injury}

The endoscopists were blinded to the prescribed regimen and assessed colonic cleansing using the Ottawa bowel preparation scale, which considers the sum of the cleanliness and the fluid quantity (0-14). A cleanliness score between 0 (no liquid) and 4 (solid stool, not washable) was rated for each of the three segments of the colon (the right colon, cecum to ascending; midcolon, transverse to descending; and the rectosigmoid colon). Fluid quantity was rated from 0 to 2 for the entire colon (minimal, 0; moderate, 1; large, 2). All participating endoscopists underwent training prior to the study to standardise their use of the Ottawa bowel preparation scale. Mucosal injuries were assessed in eight different segments (lower part of the rectum 2 $\mathrm{cm}$ above the anal verge, middle part of the sigmoid, descending colon below the splenic flexure, transverse colon just after the splenic flexure, transverse colon before the hepatic flexure, ascending colon under the hepatic flexure, ileocecal valve and cecum). Pictures of the eight segments mentioned above were taken during the insertion of the colonoscope. To avoid the misinterpretation of traumatic lesions, we compared pictures taken during the insertion with those from the retrieval of the colonoscope. If, within the same segment, mucosal injury was found in the picture taken during the retrieval but was not definitely identified in the picture taken during the insertion, we considered the mucosal injury to be due to traumatic damage. All mucosal injuries observed during the colonoscopy were noted and biopsied. Biopsied specimens were reviewed by a single pathologist who was blinded to the prescribed regimen. Mucosal abnormalities that were histologically confirmed as acute inflammatory changes were considered true mucosal injuries.

\section{Sample size and statistics}

The sample size calculation was based on comparisons of the preparation quality between the two groups. A sample size of 150 patients for each group was estimated to give an $80 \%$ power at a two-sided $\alpha$ of 0.05 for the detection of a 1.0-point difference in the Ottawa bowel preparation quality scale. We assumed a drop-out rate of $10 \%$ and aimed to enroll a total of 330 patients. To compare patient compliance, acceptance, preference to repeat the same regimen, adverse events, bowel preparation efficacy, and mucosal injury between the two arms, chi-square tests (or Fisher exact test) were used for categorical variables, whereas Student t-tests were used for continuous variables. A p-value $<0.05$ was considered statistically significant. All statistical analyses were conducted using SPSS version 15.0 (SPSS Inc., Chicago, IL, USA).

\section{RESULTS}

\section{Patient characteristics}

A total of 330 patients were selected for randomization, 11 of which were excluded after they canceled the procedures. A total of 319 patients were included in the final study, 160 of which were allocated to the SD-PEG group, and 159, to the LV$\mathrm{PEG}+$ Asc group. The mean ages were 45 and 48 years in the SD-PEG and LV-PEG+Asc groups ( $45 \pm 10.7$ vs $48 \pm 8.8, p=0.007$ ), respectively. The abdominal pelvic surgery rates were different 
between the SD-PEG and LV-PEG+Asc groups (24.4\% vs 27.2\%, $\mathrm{p}=0.044$ ). There was no significant difference in the sex, body mass index, experience of colonoscopy, family history of colon cancer, and past medical history in the two groups (Table 1).

\section{Efficacy of bowel cleansing}

There was no significant difference between the two groups for the total mean score using the Ottawa bowel preparation scale $(4.19 \pm 2.261$ vs $4.41 \pm 2.066, \mathrm{p}=0.376)$ for bowel preparation in the right colon $(1.19 \pm 0.98$ vs $1.11 \pm 0.94, \mathrm{p}=0.423)$ and the rectosigmoid colon $(1.16 \pm 0.65$ vs $1.07 \pm 0.60, p=0.221)$. However, the LV-PEG+Asc group had a greater quantity of fluid remaining in the colon $(0.66 \pm 0.623$ vs $0.81 \pm 0.542, p=0.023)$ and a worse bowel cleansing score in the middle colon compared to SD-PEG (1.19 \pm 0.940 vs $1.42 \pm 0.732, \mathrm{p}=0.014)$ (Table 2$)$.

\section{Patient affinity and adverse events}

We assessed patient compliance based on their consumption of the preparation solution and found no significant difference between the two groups (SD-PEG: 96.3\% vs LV-PEG+Asc: 96.9\%, $\mathrm{p}=0.768$ ). The LV-PEG+Asc group showed better results for both of these metrics (acceptance and preference) ( $p=0.001)$. Overall, 138 of 319 patients (43\%) experienced adverse events. The overall incidence of adverse events was not significantly different between the two groups (69/160 [43.1\%], 69/159 [43.4\%], $\mathrm{p}=0.972$ ); however, the LV-PEG+Asc group tended to had more headache and dizziness $(\mathrm{p}=0.056)$ (Table 3$)$.

\section{Mucosal injuries associated with the bowel preparation}

Mucosal inflammation/ulceration occurred in 3.1\% (5/160) of patients taking SD-PEG compared with 3.7\% (6/159) receiving LV-PEG+Asc. Using a chi-square test, the difference between

Table 1. Baseline Characteristics

\begin{tabular}{lccc}
\hline \multicolumn{1}{c}{ Characteristic } & $\begin{array}{c}\text { SD-PEG } \\
(\mathrm{n}=160)\end{array}$ & $\begin{array}{c}\text { LV-PEG+Asc } \\
(\mathrm{n}=159)\end{array}$ & $\mathrm{p}$-value* \\
\hline Age, yr & $45.0 \pm 10.7$ & $48.0 \pm 8.8$ & 0.007 \\
Male:female & $78(48.8): 82(51.2)$ & $85(53.5): 74(46.5)$ & 0.781 \\
Body mass index, $\mathrm{kg} / \mathrm{m}^{2}$ & $23.7 \pm 3.0$ & $23.6 \pm 3.1$ & 0.806 \\
Experience of colonoscopy & $76(47.5)$ & $76(47.8)$ & 0.958 \\
Previous surgical history & $39(24.4)$ & $43(27.2)$ & 0.044 \\
Abdominal operation & $17(10.6)$ & $14(8.8)$ & - \\
Pelvic or gynaecologic operation & $22(13.8)$ & $29(18.4)$ & - \\
Family history of colon cancer & $6(3.8)$ & $11(6.9)$ & 0.210 \\
Past medical history & & & 0.793 \\
Hypertension & $19(11.9)$ & $11(6.9)$ & - \\
Diabetes & $3(1.9)$ & $3(1.9)$ & - \\
Cardiovascular disease & $2(1.3)$ & $3(1.9)$ & - \\
Thyroid disease & $3(1.9)$ & $5(3.1)$ & - \\
Others & $5(3.1)$ & $12(7.5)$ & - \\
\hline
\end{tabular}

Data are presented as mean \pm SD or number $(\%)$.

SD-PEG, split-dose polyethylene glycol; LV-PEG+Asc, low-volume polyethylene glycol+ascorbic acid.

${ }^{*} \mathrm{p}<0.05$ was considered statistically significant.

Table 2. The Efficacy of Bowel Cleansing according to the Ottawa Bowel Preparation Scale (0-14)

\begin{tabular}{lccc}
\hline Location/measurement & $\begin{array}{c}\text { SD-PEG } \\
(\mathrm{n}=160)\end{array}$ & $\begin{array}{c}\text { LV-PEG+Asc } \\
(\mathrm{n}=159)\end{array}$ & $\begin{array}{c}\mathrm{p} \text {-value* } \\
\text { Right colon }\end{array}{ }^{\dagger}$ \\
Mid-colon $^{\ddagger}$ & $1.19 \pm 0.987$ & $1.11 \pm 0.945$ & 0.423 \\
Rectosigmoid colon & $1.19 \pm 0.940$ & $1.42 \pm 0.732$ & 0.014 \\
Fluid volume & $1.16 \pm 0.659$ & $1.07 \pm 0.607$ & 0.221 \\
Total score & $0.66 \pm 0.623$ & $0.81 \pm 0.542$ & 0.023 \\
\hline
\end{tabular}

Data are presented as mean \pm SD.

SD-PEG, split-dose polyethylene glycol; LV-PEG+Asc, low-volume polyethylene glycol+ascorbic acid.

${ }^{*} \mathrm{p}<0.05$ was considered statistically significant; ${ }^{\dagger}$ Right colon: cecum to ascending; ${ }^{\ddagger}$ Mid-colon: transverse to descending. 
Table 3. Patient Compliance, Preference and Acceptance, and Adverse Events

\begin{tabular}{|c|c|c|c|}
\hline Parameter & $\begin{array}{l}\text { SD-PEG } \\
(n=160)\end{array}$ & $\begin{array}{c}\text { LV-PEG+Asc } \\
\quad(n=159)\end{array}$ & p-value \\
\hline Compliance ( $\geq 75 \%$ taken dose) & $154(96.3)$ & 154 (96.9) & 0.768 \\
\hline Preference & $57(35.6)$ & $102(64.6)$ & 0.001 \\
\hline Acceptance & & & 0.001 \\
\hline 1 (easy) & $33(20.6)$ & $53(33.3)$ & \\
\hline 2 (tolerant) & $58(36.3)$ & $81(50.9)$ & \\
\hline 3 (difficult) & $69(43.1)$ & $25(15.7)$ & \\
\hline Adverse events & $69(43.1)$ & $69(43.4)$ & 0.972 \\
\hline Abdominal pain/discomfort & & & 0.391 \\
\hline Nausea/vomiting & & & 0.470 \\
\hline Headache/dizziness & & & 0.056 \\
\hline Sleep disturbance & & & 0.804 \\
\hline
\end{tabular}

Data are presented as mean \pm SD.

SD-PEG, split-dose polyethylene glycol; LV-PEG+Asc, low-volume polyethylene glycol+ascorbic acid.

* $\mathrm{p}<0.05$ was considered statistically significant.

Table 4. Mucosal Abnormalities Observed at Colonoscopy and Following Histological Analysis

\begin{tabular}{cll}
\hline Bowel preparation & \multicolumn{1}{c}{ Endoscopic findings } & \multicolumn{1}{c}{ Microscopic findings } \\
\hline SD-PEG & Aphthous ulceration of terminal ileum & Acute inflammation, moderate, with ulcer \\
& Erythema of terminal ileum & Acute inflammation, moderate, no ulcer \\
Erythema of cecum & Acute inflammation, focal, mild, no ulcer \\
Erythema of transverse colon & Acute inflammation, focal, mild, no ulcer \\
& Erythema of rectum & Acute inflammation, focal, mild, no ulcer \\
Aphthous ulceration of cecum & Acute inflammation, moderate, with ulcer \\
Aphthous ulceration of ascending colon & Acute inflammation, moderate, with ulcer \\
& Erythema of cecum & Acute inflammation, focal, mild, no ulcer \\
& Erythema of ascending colon & Acute inflammation, focal, mild, no ulcer \\
& Erythema of ascending colon & Acute inflammation, focal, mild, no ulcer \\
& Erythema of rectum & Acute inflammation, focal, mild, no ulcer \\
\hline
\end{tabular}

SD-PEG, split-dose polyethylene glycol; LV-PEG+Asc, low-volume polyethylene glycol+ascorbic acid.

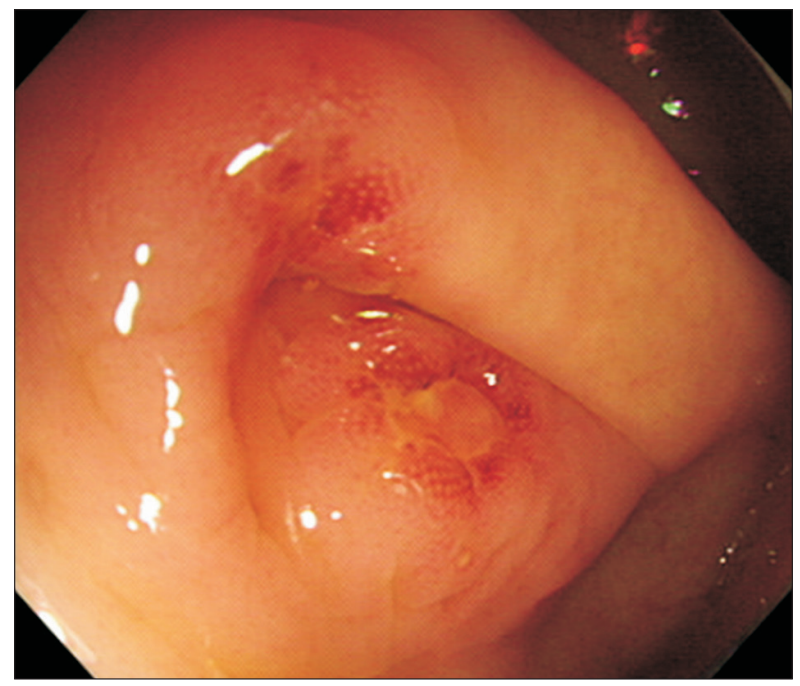

Fig. 1. Endoscopic view showing aphthous lesions in the appendiceal orifice. the two groups was not significant at an $\alpha$ value of 0.05. Endoscopically, the identified mucosal lesions that could have been associated with the two preparation regimens are reported in Table 4. Three patients were noted to have aphthous ulcers, including one patient in the SD-PEG group and two patients in the LV-PEG+Asc group (Fig. 1). Mucosal erythema was observed in eight patients. These mucosal injuries occurred in four patients from each group. The histology of all specimens confirmed acute inflammatory changes.

\section{DISCUSSION}

Proper bowel cleansing is essential for the diagnostic accuracy and therapeutic safety of colonoscopy. PEG is the commercially available regimen used most widely for bowel preparation. However, this approach requires the patient to consume a large volume of liquid over a short period of time, which some 
patients are unable to tolerate. This circumstance has led to an interest in alternative bowel cleansing regimens with better tastes and reduced volumes. Several studies have used LV-PEG with various additives, such as senna, bisacodyl, or magnesium citrate, finding an improved tolerability but a possible decrease in efficacy. ${ }^{15,16}$ Recently, a solution of LV-PEG containing Asc was developed for use before colonoscopies. Several studies have been conducted to evaluate the effectiveness and tolerability of LV-PEG+Asc compared to 4 L PEG and found that LVPEG+Asc was equally effective in colon cleansing., ${ }^{9,17}$

It has been well established that $\mathrm{NaP}$, a strong osmotic laxative, can cause colonic mucosal injuries. ${ }^{12,13}$ Colonic inflammation/ulceration has been reported in $3 \%$ to $24 \%$ of patients taking $\mathrm{NaP}$, compared with $1 \%$ to $2 \%$ of patients taking PEG. ${ }^{18}$ In addition, a larger follow-up study of 634 patients who were randomized to receive PEG, NaP, or Pico demonstrated that acute mucosal inflammation/ulceration was 10-fold greater in patients receiving $\mathrm{NaP}$ or Pico when compared with PEG. ${ }^{14} \mathrm{LV}$ $\mathrm{PEG}+$ Asc is the combination of low-dose PEG and vitamin C and can be used as an osmotic laxative. Therefore, there is a possibility for mucosal damage to the colon resulting from Asc. Still, our study's results show that acute mucosal inflammation/ ulceration did not occur significantly more with LV-PEG+Asc compared with SD-PEG. Although larger scale studies are needed, we were able to identify that LV-PEG+Asc was less likely to cause mucosal injury compared with other osmotic laxatives.

In our study, the dietary restrictions used (regular breakfast and lunch, as well as a soft diet only for dinner on the day prior to colonoscopy) were relatively liberal compared with other studies. ${ }^{19}$ Several studies have supported the use of a less restrictive diet. ${ }^{20,21}$ The dietary protocol of our study could be very feasible for outpatients due to its decreased interference in their daily lives and work schedules, resulting in an improved quality of life. We used a fixed split-dose regimen for the bowel preparations because SD-PEG regimens were associated with more appropriate bowel cleansing and better compliance than fulldose PEG regimens. ${ }^{22}$

In an attempt to avoid subjective or nonvalidated assessments of bowel cleansing, the Ottawa bowel preparation scale, an objectively framed bowel preparation quality scale, was used in this study. ${ }^{23}$ The Ottawa scale assesses colonic segments individually and colonic fluid overall to provide a summary of the entire colon. In our study, LV-PEG+Asc was as effective as SD-PEG with a similar total cleansing score $(4.19 \pm 2.261$ vs 4.41 $\pm 2.066, p=0.376)$. However, the LV-PEG+Asc group had a greater quantity of fluid remaining in the colon and worse bowel cleansing scores in the mid-colon than SD-PEG. These results were similar to those of previous studies on LV-PEG+Asc versus SD-PEG in bowel cleansing for colonoscopy. ${ }^{9,24}$ We assessed patient compliance based on their consumption of the cleansing solution. The proportion of patients who ingested at least 75\% of the cleansing solution was not significantly different be- tween the two groups, in terms of compliance (96.3\% vs 96.9\%, $\mathrm{p}=0.768$ ). This compliance was much higher in our study than in other studies. ${ }^{25,26}$ A possible explanation for this may be that our subjects were younger outpatients with a lower incidence of comorbidity compared with that of previous studies. Regarding patient acceptance and preference, the LV-PEG+Asc group showed better results; the reduced volume of PEG administered to patients may have positively influenced their responses, even in spite of their instructions to consume an additional liter of PEG-free liquid. However, patients receiving the LV-PEG+Asc preparation showed similar results in the adverse events categories, including abdominal pain/discomfort, nausea/vomiting, headache/dizziness, and sleep disturbances, when compared to SD-PEG, offering no overt advantages. One possible explanation for this finding is that the LV-PEG+Asc regimen still requires patients have to ingest a moderate to large amount of fluid over a short period of time. Further, bowel preparation agents are not always benign and can negatively impact intestinal mucosal integrity. ${ }^{18}$ It has been reported that ulcerative colitis can flare up following a colonoscopic examination. ${ }^{27}$ Previous studies have described the colonic mucosal injuries that are associated with an $\mathrm{NaP}$ regimen. ${ }^{12,28-30}$ Faigel et al. ${ }^{31}$ described a patient in whom several lesions with the appearance of aphthous ulcerations were noted after bowel preparation with PEG; such a bowel preparation may provoke a reactivation of ulcerative colitis. Cappell et $a .^{32}$ reviewed the association between medications and colonic toxicity. Various drugs, including NSAIDs, were able to cause colonic mucosal injury by impairing the ability of the mucosa to resist and respond to injury. In our study, all patients with confirmed or suspected inflammatory bowel disease and those taking NSAIDs were excluded. Our findings identified no significant difference in acute mucosal inflammation/ulceration between the LV-PEG+Asc group and the SD-PEG group.

Our study has some limitations. First, this randomized controlled trial was conducted at a single center and enrolled outpatients without significant medical problems. The results of our study require further confirmation through multicenter randomized trials, including an unselected group of patients. Second, we cannot exclude that the previous preparation may have affected the grading of the acceptance of the study's regimen. Patient who have already undergone a colonoscopy may be biased, as those aware of the SD-PEG treatment may find LV-PEG+Asc more acceptable. Enrolling patients who were undergoing colonoscopies for the first time would have been more ideal. Third, we did not control the time-to-colonoscopy interval in the two groups. Several previous studies have demonstrated that the interval between the last dose of the bowel preparation regimen and the start of the colonoscopy affects the quality of bowel preparation. ${ }^{17,33}$ However, these limitations equally affected the two groups.

In conclusion, our study showed that LV-PEG+Asc provided equal, safe and efficacious bowel cleansing compared to SD- 
PEG. LV-PEG+Asc was associated with better patient compliance and acceptance, probably because it required patients to consume a smaller volume of liquid than SD-PEG. This is the first paper to compare the acute mucosal injuries related to LVPEG+Asc and SD-PEG. There was no significant difference in the incidence and character of mucosal injury between the two groups. Based on the data, LV-PEG+Asc represent a valid alternative to SD-PEG. However, improvements are needed to reduce the adverse events encountered, and further studies should be conducted to assess patient preparation preferences.

\section{CONFLICTS OF INTEREST}

No potential conflict of interest relevant to this article was reported.

\section{REFERENCES}

1. Lin OS, Kozarek RA, Cha JM. Impact of sigmoidoscopy and colonoscopy on colorectal cancer incidence and mortality: an evidence-based review of published prospective and retrospective studies. Intest Res 2014;12:268-274.

2. Lee HS, Byeon JS. Bowel preparation, the first step for a good quality colonoscopy. Intest Res 2014;12:1-2.

3. Ell C, Fischbach W, Keller R, et al. A randomized, blinded, prospective trial to compare the safety and efficacy of three bowelcleansing solutions for colonoscopy (HSG-01*). Endoscopy 2003; 35:300-304.

4. Balaban DH, Thompson WO. Oral bowel preparation for colonoscopy. Aliment Pharmacol Ther 2007;26:965-966.

5. Aronchick CA, Lipshutz WH, Wright SH, Dufrayne F, Bergman G. A novel tableted purgative for colonoscopic preparation: efficacy and safety comparisons with Colyte and Fleet Phospho-Soda. Gastrointest Endosc 2000;52:346-352.

6. Radaelli F, Meucci G, Imperiali G, et al. High-dose senna compared with conventional PEG-ES lavage as bowel preparation for elective colonoscopy: a prospective, randomized, investigatorblinded trial. Am J Gastroenterol 2005;100:2674-2680.

7. Day LW, Velayos F. Colorectal cancer screening and surveillance in the elderly: updates and controversies. Gut Liver 2015;9:143151.

8. Regev A, Fraser G, Delpre G, et al. Comparison of two bowel preparations for colonoscopy: sodium picosulphate with magnesium citrate versus sulphate-free polyethylene glycol lavage solution. Am J Gastroenterol 1998;93:1478-1482.

9. Moon CM, Park DI, Choe YG, et al. Randomized trial of 2-L polyethylene glycol + ascorbic acid versus 4-L polyethylene glycol as bowel cleansing for colonoscopy in an optimal setting. J Gastroenterol Hepatol 2014;29:1223-1228.

10. Kim HG, Huh KC, Koo HS, et al. Sodium picosulfate with magnesium citrate (SPMC) plus laxative is a good alternative to conventional large volume polyethylene glycol in bowel preparation: a multicenter randomized single-blinded trial. Gut Liver 2015;9:494501.

11. Bae SE, Kim KJ, Eum JB, et al. A comparison of 21 of polyethylene glycol and $45 \mathrm{ml}$ of sodium phosphate versus $4 \mathrm{l}$ of polyethylene glycol for bowel cleansing: a prospective randomized trial. Gut Liver 2013;7:423-429.

12. Watts DA, Lessells AM, Penman ID, Ghosh S. Endoscopic and histologic features of sodium phosphate bowel preparation-induced colonic ulceration: case report and review. Gastrointest Endosc 2002;55:584-587.

13. Chan A, Depew W, Vanner S. Use of oral sodium phosphate colonic lavage solution by Canadian colonoscopists: pitfalls and complications. Can J Gastroenterol 1997;11:334-338.

14. Lawrance IC, Willert RP, Murray K. Bowel cleansing for colonoscopy: prospective randomized assessment of efficacy and of induced mucosal abnormality with three preparation agents. Endoscopy 2011;43:412-418.

15. Hookey LC, Depew WT, Vanner SJ. Combined low volume polyethylene glycol solution plus stimulant laxatives versus standard volume polyethylene glycol solution: a prospective, randomized study of colon cleansing before colonoscopy. Can J Gastroenterol 2006;20:101-105.

16. Sharma VK, Chockalingham SK, Ugheoke EA, et al. Prospective, randomized, controlled comparison of the use of polyethylene glycol electrolyte lavage solution in four-liter versus two-liter volumes and pretreatment with either magnesium citrate or bisacodyl for colonoscopy preparation. Gastrointest Endosc 1998;47:167171.

17. Marmo R, Rotondano G, Riccio G, et al. Effective bowel cleansing before colonoscopy: a randomized study of split-dosage versus non-split dosage regimens of high-volume versus low-volume polyethylene glycol solutions. Gastrointest Endosc 2010;72:313320.

18. Rejchrt S, Bures J, Siroky M, Kopacova M, Slezak L, Langr F. A prospective, observational study of colonic mucosal abnormalities associated with orally administered sodium phosphate for colon cleansing before colonoscopy. Gastrointest Endosc 2004;59:651654.

19. Frommer D. Cleansing ability and tolerance of three bowel preparations for colonoscopy. Dis Colon Rectum 1997;40:100-104.

20. Aoun E, Abdul-Baki H, Azar C, et al. A randomized single-blind trial of split-dose PEG-electrolyte solution without dietary restriction compared with whole dose PEG-electrolyte solution with dietary restriction for colonoscopy preparation. Gastrointest Endosc 2005;62:213-218.

21. Reilly T, Walker G. Reasons for poor colonic preparation with inpatients. Gastroenterol Nurs 2004;27:115-117.

22. Kilgore TW, Abdinoor AA, Szary NM, et al. Bowel preparation with split-dose polyethylene glycol before colonoscopy: a metaanalysis of randomized controlled trials. Gastrointest Endosc 2011; 73:1240-1245.

23. Rostom A, Jolicoeur E. Validation of a new scale for the assess- 
ment of bowel preparation quality. Gastrointest Endosc 2004;59: 482-486.

24. Valiante F, Pontone S, Hassan C, et al. A randomized controlled trial evaluating a new 2-L PEG solution plus ascorbic acid vs 4-L PEG for bowel cleansing prior to colonoscopy. Dig Liver Dis 2012; 44:224-227.

25. Cohen LB, Sanyal SM, Von Althann C, et al. Clinical trial: 2-L polyethylene glycol-based lavage solutions for colonoscopy preparation: a randomized, single-blind study of two formulations. Aliment Pharmacol Ther 2010;32:637-644.

26. Ell C, Fischbach W, Bronisch HJ, et al. Randomized trial of lowvolume PEG solution versus standard PEG + electrolytes for bowel cleansing before colonoscopy. Am J Gastroenterol 2008;103:883893.

27. Menees S, Higgins P, Korsnes S, Elta G. Does colonoscopy cause increased ulcerative colitis symptoms? Inflamm Bowel Dis 2007; 13:12-18.
28. Zwas FR, Cirillo NW, el-Serag HB, Eisen RN. Colonic mucosal abnormalities associated with oral sodium phosphate solution. Gastrointest Endosc 1996;43:463-466.

29. Driman DK, Preiksaitis HG. Colorectal inflammation and increased cell proliferation associated with oral sodium phosphate bowel preparation solution. Hum Pathol 1998;29:972-978.

30. Atkinson RJ, Save V, Hunter JO. Colonic ulceration after sodium phosphate bowel preparation. Am J Gastroenterol 2005;100:26032605.

31. Faigel DO, Furth EE, Bachwich DR. Aphthoid lesions of the rectum. Gastrointest Endosc 1996;43:528-529.

32. Cappell MS. Colonic toxicity of administered drugs and chemicals. Am J Gastroenterol 2004;99:1175-1190.

33. Siddiqui AA, Yang K, Spechler SJ, et al. Duration of the interval between the completion of bowel preparation and the start of colonoscopy predicts bowel-preparation quality. Gastrointest Endosc 2009;69:700-706. 